\title{
Redefining Cut-Points for High Symptom Burden of the Global Initiative for Chronic Obstructive Lung Disease Classification in 18,577 Patients with Chronic Obstructive Pulmonary Disease
}

Dionne E Smid, Frits ME Franssen, Maria Gonik, Marc Miravitlles, Ciro Casanova, Borja G Cosio, Pilar de Lucas-Ramos, Jose M Marin, Cristina Martinez, Isabel Mir, Joan B Soriano, Juan P de Torres, Alvar Agusti, Nart B Atalay, Julia Billington, Afroditi K Boutou, Stefanie BrighentiZogg, Emma Chaplin, Samantha Coster, James W Dodd, Selina Dürr, Alberto Fernandez-Villar, Miriam TJ Groenen, Miguel Guimarães, Karel Hejduk, Victoria Higgins, Nicholas S Hopkinson, Nobuyuki Horita, Sarah Houben-Wilke, Daisy JA Janssen, Melissa Jehn, Rudolf Joerres, Annika

Karch, Julia L Kelly, Yu-Il Kim, Hiroshi Kimura, Vladimir Koblizek, Janwillem H Kocks, Samantha SC Kon, Namhee Kwon, Inês Ladeira, Sang-Do Lee, Joerg D Leuppi, Nicholas Locantore, José L Lopez-Campos, William D-C Man, Lana Maricic, Laura Mendoza, David Miedinger, Florin Mihaltan, Seigo Minami, Thys van der Molen, Trevor J Murrells, Nienke Nakken, Yu Nishijima, Ian J Norman, Barbora Novotna, Denis E O’Donnell, Yoshitaka Ogata,

Eanes D Pereira, James Piercy, David Price, Chaicharn Pothirat, Natya Raghavan, Thomas Ringbaek, Dimitar Sajkov, Naseh Sigari, Sally Singh, Mark Small, Guilherme F da Silva, Rebecca J Tanner, Ioanna G Tsiligianni, Baykal Tulek, Nikolaos Tzanakis, Lowie EGW Vanfleteren, Henrik Watz, Katherine A Webb, Emiel FM Wouters, Guogang G Xie, Masanori Yoshikawa and Martijn A Spruit 


\section{ABSTRACT}

Background Patients with chronic obstructive pulmonary disease (COPD) can be classified into groups $\mathrm{A} / \mathrm{C}$ or $\mathrm{B} / \mathrm{D}$ based on symptom intensity. Different threshold values for symptom questionnaires can result in misclassification and, in turn, different treatment recommendations. The primary aim was to find the best fitting cut-points for Global initiative for chronic Obstructive Lung Disease (GOLD) symptom measures, with a modified Medical Research Council dyspnea grade of 2 or higher as point of reference.

Methods After a computerized search, data from 41 cohorts and whose authors agreed to provide data were pooled. COPD studies were eligible for analyses if they included, at least age, sex, postbronchodilator spirometry, modified Medical Research Council, and COPD Assessment Test (CAT) total scores.

Main outcomes Receiver operating characteristic curves and the Youden index were used to determine the best calibration threshold for CAT, COPD Clinical Questionnaire, and St. Georges Respiratory Questionnaire total scores. Following, GOLD A/B/C/D frequencies were calculated based on current cut-points and the newly derived cut-points.

Findings A total of 18,577 patients with COPD [72.0\% male; mean age: 66.3 years (standard deviation 9.6)] were analyzed. Most patients had a moderate or severe degree of airflow limitation (GOLD spirometric grade 1, 10.9\%; grade 2, 46.6\%; grade 3, 32.4\%; and grade 4, 10.3\%). The best calibration threshold for CAT total score was 18 points, for COPD Clinical Questionnaire total score 1.9 points, and for St. Georges Respiratory Questionnaire total score 46.0 points.

Conclusions The application of these new cut-points would reclassify about one-third of the patients with COPD and, thus, would impact on individual disease management. Further validation in prospective studies of these new values are needed. 


\section{INTRODUCTION}

The Global initiative for chronic Obstructive Lung Disease (GOLD) is a recent practice strategy on the diagnosis, prevention, and management of chronic obstructive pulmonary disease (COPD) (1). Patients with COPD are classified based on postbronchodilator spirometry into grade I (forced expiratory volume in the first second, $\mathrm{FEV}_{1} \geq 80 \%$ predicted), grade II ( $\mathrm{FEV}_{1} 50 \%$ to $<80 \%$ predicted), grade III ( $\mathrm{FEV}_{1} 30 \%$ to $<50 \%$ predicted), or grade IV (FEV $<30 \%$ predicted). Additionally, patients are classified in groups A to D for specific therapeutic recommendations based on the degree of symptoms (low vs high), and the history of exacerbations and hospitalizations.

High symptoms are determined using various questionnaires: the modified Medical Research Council scale (mMRC, grade 2 or higher), the COPD Clinical Questionnaire (CCQ) total score (1 point or higher), the COPD Assessment test (CAT) total score (10 points or higher), and the St. Georges Respiratory Questionnaire (SGRQ) total score (25 points or higher) (1).

The choice of symptom questionnaire impacts the classification of patients with COPD in gold A/C OR B/D groups (2-5). mMRC only focusses on the impact of dyspnea, the most common symptom of patients with COPD. The other symptom questionnaires also take other aspects of health-related quality of life into consideration. Therefore, the CAT, CCQ, or SGRQ may be preferred over MMRC. However, mMRC is easy to obtain, and, it is the most frequently used in clinical practice and studies, and is suggested to be used to categorize patients into symptom severity groups for the purpose of treatment $(1,6)$.

Previous studies suggest that the current cut-points need proper validation (4,7-10). Indeed, the GOLD Scientific Committee recognized that a calibration of the current cut-points of the symptom measures is an important topic that needs to be addressed in the next major revision of the gold document (10). Then again, the former threshold values for mMRC, SGRQ, and CAT are still used in the GOLD 2017 strategy.1 Interestingly, the GOLD document indicates that multidimensional scores like CAT do not categorize patients into symptom severity groups for the purpose of treatment, suggesting a central role of mMRC for patient classification. Therefore, new classification schemes should be benchmarked against mMRC.

The primary aim of this patient-level pooled analysis was to find best fitting cut-points for GOLD symptom measures, with a mMRC dyspnea grade of 2 or higher as the point of reference. Following, the impact of the newly derived cut-points of all questionnaires on the frequency distribution of the GOLD staging was studied. 


\section{METHODS}

This is a pooled analysis of concurrent cohort studies assessing mMRC and multidimensional evaluation systems in COPD. To identify the original cohorts, we performed a computerized search in the database Medline/Pubmed for reports published from the first CAT publication (September 2009) to June 2015 (11). D.S., S.H-W., or M.S. approached the corresponding authors to gather information about their readiness to partake and the availability of a minimum required set of individual data of patients with COPD, including age, sex, postbronchodilator $\mathrm{FEV}_{1}$, CAT total score, and mMRC dyspnea grade. All participants within their respective studies gave their informed written consent to participate in the original study, and each study was approved by their respective ethics committee.

\section{Measurements}

The individual demographics and clinical characteristics [sex, age (years), height (m), weight (kg), smoking status (current/former/never), pack years, use of long-term oxygen therapy (yes/no), $\mathrm{FEV}_{1}$ (liters), $\mathrm{FEV}_{1}\left(\%\right.$ predicted), $\mathrm{FEV}_{1} / \mathrm{FVC}(\%)$, mMRC dyspnea grade, CAT total score (points), CCQ total score (points), SGRQ total score (points), and number of COPD exacerbations and/or hospitalizations in the last 12 months] were provided from each dataset. All data were pooled, and the dataset was cleaned.

\section{Statistics}

Mean (standard deviation), median (interquartile range), or proportions were calculated, whatever appropriate. Pearson product moment correlations between mMRC dyspnea grade, CAT total score, CCQ total score, and SGRQ total score were performed. A r-value of $< \pm 0.20$ indicates no meaningful correlation; \pm 0.20 to \pm 0.34 , weak; \pm 0.35 to \pm 0.50 , moderate; and $> \pm 0.50$, strong correlation (12). Postbronchodilator FEV $\mathrm{F}_{1}$ was used to classify patients into spirometric grades 1 4. Allocation to GOLD groups A-D was done using $\mathrm{mMRC} \geq 2$, CAT $\geq 10$, CCQ $\geq 1$, and SGRQ $\geq 25$ in combination with exacerbations history. In addition, patients were re-allocated (if applicable) based on the newly derived cut-points in combination with exacerbations history.

Receiver operating characteristic (ROC) curves were used to reveal the cut-points for the GOLD symptom measures that discriminate best between the 2 clusters defined on mMRC dyspnea grade ( 2 or higher). ROC curve represented dependency between the sensitivity and specificity of the binary classification for different cut-points of the GOLD symptom measures. The cut-point, which satisfied the optimal criterion of the Youden index (13), was referred as the best calibration threshold. The optimal cut-points were calculated for CAT total score, CCQ total score, and SGRQ total score. A software environment $\mathrm{R}$ v 3.1.0 was used. The ROC function from the pROC package was used to visualize the ROC curves and calculate the best thresholds.

Graphs were created using GraphPad Prism v 6 (GraphPad Software Inc. San Diego, CA). Statistics were performed using SPSS for Windows, v 20.0 (IBM Corp, Armonk, NY). A P value of $\leq .01$ was interpreted as statistically significant, to obtain a greater statistical power than the usual $P$ value of $<.05$. 


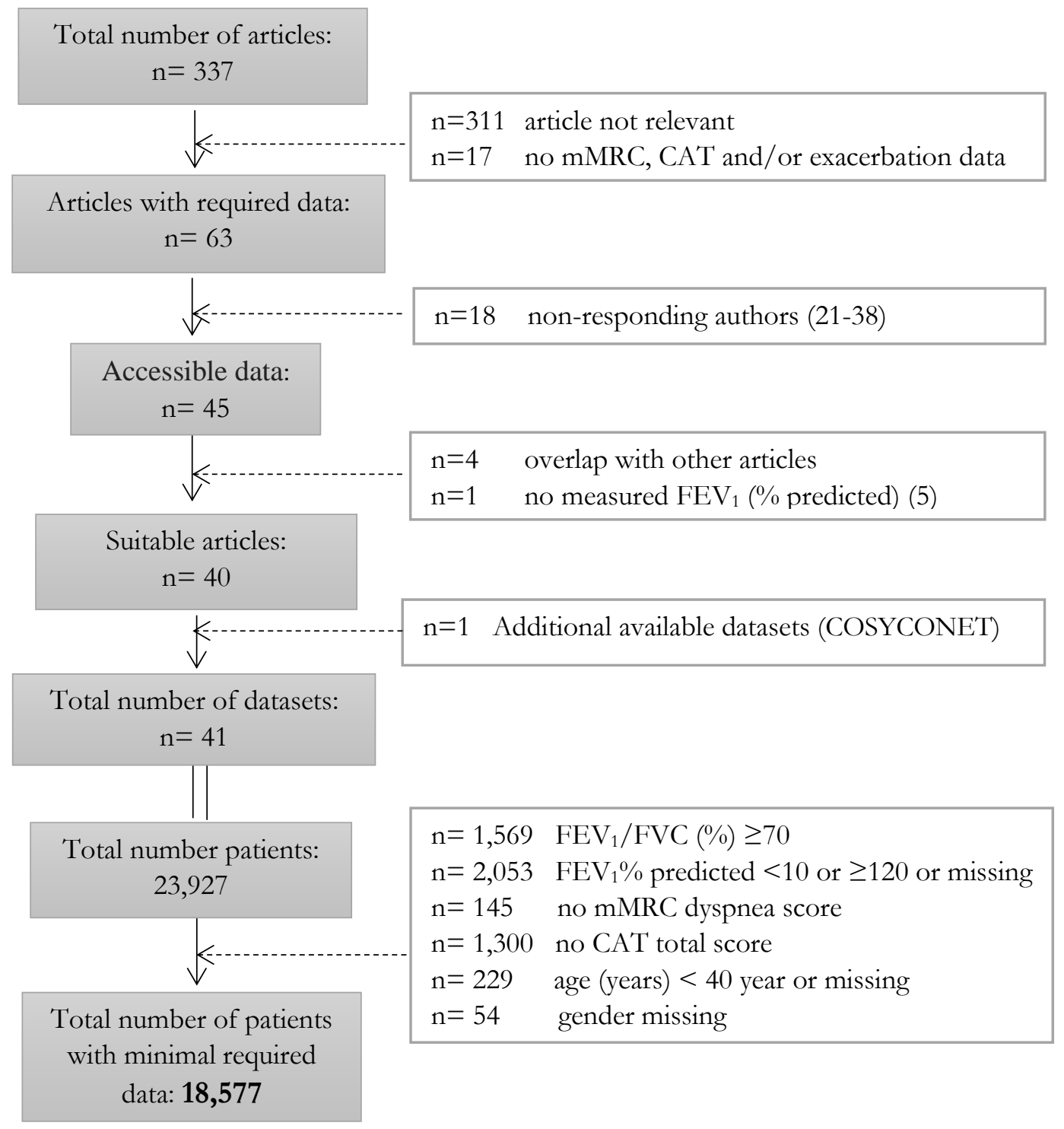

Figure E1. Flow diagram of subject inclusion 
Table 1. General characteristics per resource article

\begin{tabular}{|c|c|c|c|c|c|c|c|c|c|c|c|c|}
\hline \multirow[b]{2}{*}{ Lead author } & \multicolumn{12}{|c|}{ Dataset resource } \\
\hline & $\begin{array}{r}\text { Agusti } \\
\text { (39) }\end{array}$ & $\begin{array}{r}\text { Billington } \\
(40)\end{array}$ & $\begin{array}{r}\text { Boutou } \\
(41)\end{array}$ & $\begin{array}{r}\text { De Torres } \\
(42)\end{array}$ & $\begin{array}{r}\text { Casanova } \\
(43)\end{array}$ & $\begin{array}{r}\text { Casanova } \\
(43)\end{array}$ & $\begin{array}{r}\text { Chaplin } \\
\text { (44) }\end{array}$ & $\begin{array}{r}\text { Dodd } \\
(45)\end{array}$ & $\begin{array}{r}\text { Horita } \\
(46)\end{array}$ & $\begin{array}{r}\text { Jehn } \\
(47)\end{array}$ & $\begin{array}{r}\text { Jones } \\
(2)\end{array}$ & $\begin{array}{r}\text { Karch } \\
(20)\end{array}$ \\
\hline Journal and year & $\begin{array}{r}\text { Qual Life Res, } \\
2015\end{array}$ & COPD, 2015 & $\begin{array}{r}\text { BMJ Open Respir } \\
\text { Res, } 2014\end{array}$ & $\begin{array}{r}\text { CHEST, } \\
2014\end{array}$ & $\begin{array}{r}\text { Respir } \\
\text { Res, } 2014\end{array}$ & $\begin{array}{r}\text { Chest, } \\
2014\end{array}$ & $\begin{array}{r}\text { J Cardiopulm } \\
\text { Rehabil Prev, } 2015\end{array}$ & $\begin{array}{r}\text { Thorax, } \\
2011\end{array}$ & $\begin{array}{r}\text { Clin Respir } \\
\mathrm{J}, 2013\end{array}$ & $\begin{array}{r}\text { Environ Health, } \\
2013\end{array}$ & ERJ, 2013 & Respir Med, 2016 \\
\hline Country & Spain & UK & UK & & & Spain & UK & UK & Japan & Germany & $\begin{array}{l}\text { Belgium, France, UK, } \\
\text { Germany, Italy, the } \\
\text { Netherlands and Spain }\end{array}$ & Germany \\
\hline Cohort & GSK & & & & & CHAIN & & & & & HEED & COSYCONET \\
\hline $\mathbf{n}$ & 110 & 61 & 703 & & & 785 & 166 & 196 & 74 & 34 & 1725 & 2258 \\
\hline Gender (male), n (\%) & $104(94.5)$ & $29(47.5)$ & $348(49.5)$ & & & $658(83.8)$ & $89(53.6)$ & $114(58.2)$ & $60(81.1)$ & $22(64.7)$ & $1251(72.5)$ & $1379(61.1)$ \\
\hline Age, years & $70.3(9.7)$ & $71.2(10.0)$ & $67.3(9.8)$ & & & $67.8(8.8)$ & $70.8(8.7)$ & $69.2(9.0)$ & $72.3(9.4)$ & $63.5(9.9)$ & $64.9(9.7)$ & $65.1(8.4)$ \\
\hline Current smoker, n (\%) & $28(25.5)$ & $32(52.5)$ & - & & & $223(28.4)$ & - & - & $11(14.9)$ & $28(82.4)$ & $737(42.7)$ & $561(24.8)$ \\
\hline Packyears & $40.0(24.6-54.3)^{\mathrm{a}}$ & - & - & & & $55.9(28.0)^{\mathrm{h}}$ & - & - & $51.6(30.4)$ & $15.7(12.1)$ & $38.4(19.2)^{\mathrm{p}}$ & $41.2(22.0-63.0)^{v}$ \\
\hline BMI, $\mathrm{kg} / \mathrm{m}^{2}$ & - & $26.1(22.3-29.9)$ & $26.0(22.4-30.0)^{\mathrm{d}}$ & & 27.8 & $(24.8-31.0)^{\mathrm{i}}$ & $27.7(7.0)^{\mathrm{k}}$ & $27.6(6.6)^{1}$ & $21.2(3.0)$ & $26.5(19.9-29.8)$ & $27.0(4.9)^{\mathrm{q}}$ & $26.2(23.2-29.4)^{\mathrm{w}}$ \\
\hline FEV $_{1}(\%$ pred.) & $63.6(20.0)$ & $65.4(15.8)$ & $49.2(18.8)$ & & & $59.3(20.2)$ & $56.2(24.4)$ & $51.2(19.4)$ & $43.2(12.7)$ & $46.2(13.4)$ & $56.4(19.7)$ & $52.5(18.5)$ \\
\hline $\mathrm{FEV}_{1} / \mathrm{FVC}(\%)$ & $54.0(11.2)^{\mathrm{b}}$ & $53.0(10.2)$ & $43.9(14.5)^{\mathrm{e}}$ & & & $51.9(11.3)$ & - & - & $59.7(7.7)$ & $42.3(13.2)^{\circ}$ & $56.2(10.6)^{r}$ & $51.3(11.0)$ \\
\hline LTOT, n (\%) & - & - & & & & $69(22.3)^{j}$ & - & - & $16(21.6)$ & $8(23.5)$ & - & $436(19.3)$ \\
\hline GOLD stage, $n(\%)-I$ & $24(21.8)$ & $12(19.7)$ & $45(6.4)$ & & & $134(17.1)$ & $35(21.1)$ & $16(8.2)$ & $0(0.0)$ & $0(0.0)$ & $216(12.5)$ & $202(8.9)$ \\
\hline- II & $57(51.8)$ & $39(63.9)$ & $289(41.1)$ & & & $377(48.0)$ & $55(33.1)$ & $86(43.9)$ & $20(27.0)$ & $14(41.2)$ & $827(47.9)$ & $956(42.3)$ \\
\hline - III & $24(21.8)$ & $9(14.8)$ & $246(35.0)$ & & & $231(29.4)$ & $50(30.1)$ & $65(33.2)$ & $42(56.8)$ & $15(44.1)$ & $540(31.3)$ & $856(37.9)$ \\
\hline - IV & $5(4.5)$ & $1(1.6)$ & $123(17.5)$ & & & $43(5.5)$ & $26(15.7)$ & $29(14.8)$ & $12(16.2)$ & $5(14.7)$ & $142(8.2)$ & $244(10.8)$ \\
\hline $\begin{array}{l}\text { Exacerbations previous } \\
12 \text { months } \geq 2, n(\%)\end{array}$ & $9(8.2)$ & $41(67.2)$ & - & & & $98(12.5)$ & - & - & - & $11(32.4)$ & $451(29.9)^{\mathrm{s}}$ & $633(28.0)$ \\
\hline $\begin{array}{l}\text { Hospitalizations previous } \\
12 \text { months } \geq 1, \text { n (\%) }\end{array}$ & $6(5.5)$ & - & - & & & $89(11.3)$ & - & - & - & $20(58.8)$ & $155(10.3)^{\mathrm{t}}$ & $453(20.1)^{x}$ \\
\hline $\begin{array}{l}\text { mMRC dyspnea grade } \\
\geq 2, \mathrm{n}(\%)\end{array}$ & $49(44.5)$ & $41(67.2)$ & $576(81.9)$ & & & $358(45.6)$ & $131(78.9)$ & $148(75.5)$ & $35(47.3)$ & $16(47.1)$ & $751(43.5)$ & $1090(48.3)$ \\
\hline CAT total score, points & $16.3(8.2)$ & $14.8(6.8)$ & $21.2(7.5)$ & & & $12.1(7.6)$ & $21.8(7.6)$ & $20.2(7.5)$ & $11.1(7.9)$ & $19.1(5.7)$ & $17.7(8.4)$ & $18.1(7.4)$ \\
\hline CCQ total score, points & - & - & $3.1(1.2)^{\mathrm{f}}$ & & & $1.6(1.1)$ & - & $2.9(1.2)^{\mathrm{m}}$ & - & - & - & - \\
\hline SGRQ total score, points & $44.5(24.1)^{\mathrm{c}}$ & - & $47.3(16.4)^{g}$ & & & - & - & $45.7(19.1)^{\mathrm{n}}$ & - & - & $44.7(19.4)^{\mathrm{u}}$ & $43.6(19.9)^{y}$ \\
\hline
\end{tabular}

Values expressed as mean (SD), median (IQR) or number of patients (\%). Abbreviations: BMI= body mass index; CAT= COPD Assessment Test; CCQ= Clinical COPD Questionnaire; $\mathrm{FEV}_{1}=$ Forced Expiratory Volume in the first second; $\mathrm{FVC}=$ Forced Vital Capacity; $\mathrm{GOLD}=$ Global initiative for chronic Obstructive Lung Disease; LTOT= long-term oxygen therapy; mMRC= modified Medical Research Council dyspnoea scale; SGRQ= St. George's Respiratory Questionnaire. ${ }^{a} 8$ missing; ${ }^{b} 1$ missing; ${ }^{\mathrm{c}} 7$ missing; ${ }^{\mathrm{d}} 190$ missing; ${ }^{\mathrm{e}} 462$ missing; ${ }^{\mathrm{f}} 522$ missing; ${ }^{\mathrm{g}} 495 \mathrm{missing} ;{ }^{\mathrm{h}} 11 \mathrm{missing} ;{ }^{\mathrm{i}} 4 \mathrm{missing} ;{ }^{\mathrm{j}} 474 \mathrm{missing} ;{ }^{\mathrm{k}} 25 \mathrm{missing} ;{ }^{1} 18 \mathrm{missing} ;{ }^{\mathrm{m}} 159 \mathrm{missing} ;{ }^{\mathrm{n}} 157 \mathrm{missing} ;{ }^{\circ} 2 \mathrm{missing}$; ${ }^{\mathrm{p}} 71$ missing; ${ }^{\mathrm{q}} 6$ missing; ${ }^{\mathrm{r}} 49$ missing; ${ }^{\mathrm{s}} 151$ missing; ${ }^{\mathrm{t}} 218 \mathrm{missing} ;{ }^{\mathrm{u}} 151$ missing; ${ }^{\mathrm{v}} 11$ missing; ${ }^{\mathrm{w}} 2 \mathrm{missing} ;{ }^{\mathrm{x}} 2$ missing; ${ }^{\mathrm{y}} 12$ missing. 


\begin{tabular}{|c|c|c|c|c|c|c|c|c|c|c|c|}
\hline \multicolumn{12}{|c|}{ Dataset resource } \\
\hline Author & $\begin{array}{r}\text { Kelly } \\
(48)\end{array}$ & $\begin{array}{l}\text { Kim } \\
(49)\end{array}$ & $\begin{array}{l}\text { Kon } \\
(50)\end{array}$ & $\begin{array}{r}\text { Kwon } \\
(51)\end{array}$ & $\begin{array}{l}\text { Lee } \\
(52)\end{array}$ & $\begin{array}{r}\text { Ladeira } \\
(53)\end{array}$ & $\begin{array}{r}\text { Lopez-Campos } \\
(54)\end{array}$ & $\begin{array}{r}\text { Manca } \\
(55)\end{array}$ & $\begin{array}{r}\text { Maricic } \\
(56)\end{array}$ & $\begin{array}{r}\text { Mendoza } \\
\text { (57) }\end{array}$ & $\begin{array}{r}\text { Mihaltan } \\
(58)\end{array}$ \\
\hline $\begin{array}{lll}\begin{array}{l}\text { Journal } \\
\text { published }\end{array} & \text { and } & \text { year } \\
\end{array}$ & $\begin{array}{r}\text { Respiration, } \\
2012\end{array}$ & $\begin{array}{r}\text { Pulm Med, } \\
2013\end{array}$ & Thorax, 2014 & CHEST, 2013 & Respir Med, 2014 & $\begin{array}{r}\text { Rev Port Pneumol, } \\
2015\end{array}$ & $\begin{array}{r}\text { Int J COPD, } \\
2015\end{array}$ & COPD, 2014 & $\begin{array}{r}\text { Coll Antropol, } \\
2013\end{array}$ & $\begin{array}{r}\text { Eur Respir J, } \\
2015\end{array}$ & $\begin{array}{r}\text { Pneumologia, } \\
2015\end{array}$ \\
\hline Country & UK & South Korea & the UK & $\begin{array}{r}\text { Indonesia, Korea, } \\
\text { Vietnam and Hong } \\
\text { Kong }\end{array}$ & $\begin{array}{r}\text { Australia, China, } \\
\text { Korea and Taiwan }\end{array}$ & Portugal & Spain & Spain & Croatia & Chile & Romania \\
\hline Cohort & & - & - & GSK & GSK & & On-Sint & & & & \\
\hline $\mathbf{n}$ & 219 & 238 & 260 & 303 & 321 & 82 & 499 & 92 & 33 & 101 & 1082 \\
\hline Gender (male), n (\%) & $139(63.5)$ & $192(80.7)$ & $151(58.1)$ & $296(97.7)$ & $286(89.1)$ & $64(78.0)$ & 407 (81.6) & $67(72.8)$ & $25(75.8)$ & $62(61.4)$ & $801(74.0)$ \\
\hline Age, years & $64.0(9.6)$ & $67.8(9.4)$ & $71.0(8.8)$ & $69.0(9.4)$ & $69.7(8.8)$ & $70.2(9.5)$ & $67.1(9.3)$ & $66.1(10.8)$ & $62.6(7.9)$ & $68.8(8.5)$ & $63.1(10.1)$ \\
\hline Current smoker, n (\%) & $28(12.8)$ & $51(21.4)$ & $31(11.9)$ & $53(17.5)$ & $62(19.3)$ & $17(20.7)$ & $115(23.0)^{\mathrm{f}}$ & $4(4.3)$ & $12(36.4)^{1}$ & - & $569(52.6)$ \\
\hline Packyears & $41.7(23.2)^{\mathrm{a}}$ & $27.9(26.2)$ & $41.1(29.5)$ & $30.0(20.0-50.0)$ & $40.0(25.0-60.0)$ & $41.5(20.0-75.0)$ & $38.3(20.6)$ & $33.0(15.5-60.0)$ & $41.6(35.2)$ & $40.5(20.6)$ & - \\
\hline BMI, $\mathrm{kg} / \mathrm{m}^{2}$ & $25.2(5.4)^{\mathrm{b}}$ & $22.9(3.2)^{\mathrm{c}}$ & $27.0(23.7-31.0)$ & $20.8(3.6)$ & $23.4(4.1)$ & $26.2(5.0)$ & $27.7(4.3)^{\mathrm{g}}$ & $26.2(4.7)$ & $24.5(3.5)$ & $26.9(4.5)$ & - \\
\hline FEV $_{1}$ (\% pred.) & $40.4(17.9)$ & $72.4(23.7)$ & $50.1(20.6)$ & $49.9(18.0)$ & $50.6(19.3)$ & $44.8(16.3)$ & $58.7(18.4)$ & $48.6(17.2)$ & $67.5(16.9)$ & $66.1(19.5)$ & $56.6(17.8)$ \\
\hline $\mathrm{FEV}_{1} / \mathrm{FVC}(\%)$ & $36.9(12.7)$ & $54.5(11.8)$ & $50.4(14.3)$ & $50.0(9.8)$ & $46.5(12.1)$ & $67.0(11.4)$ & $54.6(10.8)^{\mathrm{h}}$ & $47.3(11.6)$ & $57.3(7.9)$ & $55.0(9.5)$ & - \\
\hline LTOT, n (\%) & $32(14.6)$ & - & $12(4.6)$ & - & - & $47(57.3)$ & $69(14.2)^{\mathrm{i}}$ & $7(7.6)$ & - & $3(3.0)$ & - \\
\hline GOLD stage, n (\%) - I & $8(3.7)$ & $99(41.6)$ & $26(10.0)$ & $18(5.9)$ & $25(7.8)$ & $2(2.4)$ & $55(11.0)$ & $5(5.4)$ & $6(18.2)$ & $28(27.7)$ & $119(11.0)$ \\
\hline - II & $45(20.5)$ & $92(38.7)$ & $96(36.9)$ & $131(43.2)$ & $121(37.7)$ & $22(26.8)$ & $304(60.9)$ & $32(34.8)$ & $24(72.7)$ & $52(51.5)$ & $560(51.8)$ \\
\hline - III & $96(43.8)$ & $38(16.0)$ & $84(32.3)$ & $121(39.9)$ & $137(42.7)$ & $48(58.5)$ & $112(22.4)$ & $45(48.9)$ & $2(6.1)$ & $18(17.8)$ & $336(31.1)$ \\
\hline- IV & $70(32.0)$ & $9(3.8)$ & $54(20.8)$ & $33(10.9)$ & $38(11.8)$ & $10(12.2)$ & $28(5.6)$ & $10(10.9)$ & $1(3.0)$ & $3(3.0)$ & $67(6.2)$ \\
\hline $\begin{array}{l}\text { Exacerbations previous } \\
12 \text { months } \geq 2, \mathrm{n}(\%) \\
\end{array}$ & $132(60.3)$ & $126(52.9)$ & $117(45.0)$ & $95(32.1)^{\mathrm{d}}$ & $153(47.7)$ & $26(31.7)$ & $309(62.6)^{j}$ & $29(31.5)$ & - & - & $470(43.4)$ \\
\hline $\begin{array}{l}\text { Hospitalizations previous } \\
12 \text { months } \geq 1 \text {, n (\%) }\end{array}$ & - & - & - & & - & $25(30.5)$ & $161(33.8)^{\mathrm{k}}$ & $15(16.3)$ & - & - & \\
\hline $\begin{array}{l}\text { mMRC dyspnea grade } \\
\geq 2, \mathrm{n}(\%)\end{array}$ & $183(83.6)$ & $93(39.1)$ & $185(71.2)$ & $169(55.8)$ & $153(47.7)$ & $54(65.9)$ & $247(49.5)$ & $51(55.4)$ & $6(18.2)$ & $49(48.5)$ & $665(61.5)$ \\
\hline CAT total score, points & $23.1(8.1)$ & $16.0(9.3)$ & $20.7(7.9)$ & $17.8(8.1)$ & $14.8(8.0)$ & $17.3(8.2)$ & $18.4(7.6)$ & $12.8(8.1)$ & $14.6(7.7)$ & $16.0(8.2)$ & $17.8(7.9)$ \\
\hline CCQ total score, points & - & - & $2.8(1.2)$ & - & - & - & - & - & - & - & - \\
\hline SGRQ total score, points & - & - & $49.3(16.4)$ & $45.4(17.8)^{\mathrm{e}}$ & - & - & - & - & - & $42.8(18.4)$ & - \\
\hline
\end{tabular}

Values expressed as mean (SD), median (IQR) or number of patients (\%). Abbreviations: BMI= body mass index; CAT= COPD Assessment Test; CCQ= Clinical COPD Questionnaire; $\mathrm{FEV}_{1}=$ Forced Expiratory Volume in the first second; FVC= Forced Vital Capacity; GOLD= Global initiative for chronic Obstructive Lung Disease; LTOT= long-term oxygen therapy; mMRC = modified Medical Research Council dyspnoea scale; SGRQ= St. George's Respiratory Questionnaire. ${ }^{\text {a }} 125$ missing; ${ }^{\mathrm{b}} 8$ missing; ${ }^{\mathrm{c}} 3$ missing; ${ }^{\mathrm{d}} 7$ missing; ${ }^{\mathrm{e}} 1$ missing; ${ }^{\mathrm{f}} 3$ missing; ${ }^{\mathrm{g}} 6$ missing; ${ }^{\mathrm{h}} 125$ missing; ${ }^{\mathrm{i}} 14$ missing; ${ }^{\mathrm{j}} 5 \mathrm{missing} ;{ }^{\mathrm{k}} 23 \mathrm{missing} ;{ }^{1} 21$ missing. 


\begin{tabular}{|c|c|c|c|c|c|c|c|c|c|c|}
\hline \multicolumn{11}{|c|}{ Dataset resource } \\
\hline Author & $\begin{array}{r}\text { Miravitlles } \\
(59)\end{array}$ & $\begin{array}{r}\text { Miravitlles } \\
(60)\end{array}$ & $\begin{array}{r}\text { Minami } \\
(61)\end{array}$ & $\begin{array}{r}\text { Nakken } \\
(62)\end{array}$ & $\begin{array}{r}\text { Nishijima } \\
(63)\end{array}$ & $\begin{array}{r}\text { Novotna } \\
(64)\end{array}$ & $\begin{array}{r}\text { Pothirat } \\
(65)\end{array}$ & $\begin{array}{r}\text { Pothirat } \\
(66)\end{array}$ & $\begin{array}{r}\text { Price } \\
(67)\end{array}$ & $\begin{array}{r}\text { Raghavan } \\
\text { (68) }\end{array}$ \\
\hline $\begin{array}{lll}\begin{array}{l}\text { Journal } \\
\text { published }\end{array} & \text { and } & \text { year } \\
\end{array}$ & IJTLD, 2015 & $\begin{array}{r}\text { Respir Med, } \\
2014\end{array}$ & $\begin{array}{r}\text { Multidiscip } \\
\text { Respir Med, } 2014\end{array}$ & $\begin{array}{r}\text { BMJ Open, } \\
2014\end{array}$ & $\begin{array}{r}\text { Int J Chron } \\
\text { Obstruct Pulmon } \\
\text { Dis, } 2015\end{array}$ & IJCOPD, 2014 & $\begin{array}{r}\text { BMC Pulm Med, } \\
2014\end{array}$ & $\begin{array}{r}\text { Int J Chron } \\
\text { Obstruct Pulmon } \\
\text { Dis, } 2015\end{array}$ & Int J COPD, 2014 & COPD, 2012 \\
\hline Country & Spain & Spain & Japan & $\begin{array}{r}\text { The } \\
\text { Netherlands }\end{array}$ & Japan & The Czech republic & Thailand & Thailand & $\begin{array}{l}\text { US, France, Spain, } \\
\text { Germany, Italy, UK }\end{array}$ & Canada \\
\hline Cohort & INSEPOC study & & & & & $\begin{array}{r}\text { Czech Multicentre } \\
\text { Research Database of } \\
\text { COPD (CMRD) }\end{array}$ & & & $\begin{array}{r}\text { Adelphi Respiratory } \\
\text { DSP }\end{array}$ & COLD \\
\hline $\mathbf{n}$ & 2721 & 696 & 50 & 193 & 16 & 514 & 97 & 153 & 1070 & 111 \\
\hline Gender (male), n (\%) & $2251(82.7)$ & $585(84.1)$ & $47(94.0)$ & $101(52.3)$ & $15(93.8)$ & $374(72.8)$ & $80(82.5)$ & $89(58.2)$ & $734(68.6)$ & $63(56.8)$ \\
\hline Age, years & $66.9(9.7)$ & $68.7(9.3)$ & $71.0(8.9)$ & $66.0(8.7)$ & $73.5(6.6)$ & $67.3(8.1)$ & $70.7(8.2)$ & $71.5(8.5)$ & $64.6(10.4)$ & $64.3(10.6)$ \\
\hline Current smoker, n (\%) & $1959(72.0)^{\mathrm{a}}$ & $156(22.4)^{\mathrm{f}}$ & $11(22.0)$ & $32(16.6)$ & $2(12.5)$ & $93(18.1)$ & $5(5.2)$ & - & $338(31.6)$ & $21(18.9)$ \\
\hline Packyears & $36.0(24.0-50.0)^{\mathrm{b}}$ & $43.2(21.6)^{g}$ & $63.5(32.2)$ & $37.8(28.3-51.4)$ & $37.5(24.6)^{\mathrm{m}}$ & $38.0(25.0-48.0)^{\mathrm{n}}$ & $34.0(19.0-59.5)$ & - & $32.0(20.0-48.0)^{\mathrm{q}}$ & $19.6(22.6)^{\mathrm{u}}$ \\
\hline BMI, $\mathrm{kg} / \mathrm{m}^{2}$ & $27.7(4.3)^{\mathrm{c}}$ & $26.8(24.7-30.0)^{\mathrm{h}}$ & $22.9(3.8)$ & $26.3(5.3)$ & $20.6(2.4)$ & $27.2(5.9)$ & $20.9(3.5)$ & $20.2(3.9)$ & $26.4(23.4-29.4)^{\mathrm{r}}$ & $27.2(24.3-30.8)$ \\
\hline $\mathrm{FEV}_{1}(\%$ pred.) & $52.6(18.9)$ & $53.2(19.6)$ & $51.5(18.9)$ & $47.3(17.7)$ & $54.8(18.5)$ & $43.3(11.2)$ & $56.4(21.0)$ & $47.8(17.6)$ & $60.5(16.0)$ & $86.7(15.8)$ \\
\hline $\mathrm{FEV}_{1} / \mathrm{FVC}(\%)$ & $53.4(11.1)^{\mathrm{d}}$ & $53.1(12.1)^{\mathrm{i}}$ & $59.3(9.5)$ & $40.3(12.7)$ & $59.6(9.6)$ & $50.5(10.7)$ & $50.8(12.1)$ & $51.9(10.3)$ & - & $65.0(60.0-67.6)$ \\
\hline LTOT, n (\%) & $302(11.8)^{\mathrm{e}}$ & $163(24.4)^{\mathrm{j}}$ & $0(0.0)$ & $53(27.5)$ & $0(0.0)$ & $78(15.2)$ & - & - & $143(13.4)$ & $0(0.0)$ \\
\hline GOLD stage, $\mathbf{n}(\%)-I$ & $232(8.5)$ & $46(6.6)$ & $4(8.0)$ & $0(0.0)$ & $0(0.0)$ & $0(0.0)$ & $14(14.4)$ & $10(6.5)$ & $112(10.5)$ & $77(69.4)$ \\
\hline - II & $1202(44.2)$ & $347(49.9)$ & $22(44.0)$ & $93(48.2)$ & $10(62.5)$ & $168(32.7)$ & $42(43.3)$ & $58(37.9)$ & $711(66.4)$ & $32(28.8)$ \\
\hline - III & $984(36.2)$ & $229(32.9)$ & $17(34.0)$ & $62(32.1)$ & $3(18.8)$ & $278(54.1)$ & $27(27.8)$ & $61(39.9)$ & $203(19.0)$ & $2(1.8)$ \\
\hline - IV & $303(11.1)$ & 74 (10.6) & $7(14.0)$ & 38 (19.7) & $3(18.8)$ & $68(13.2)$ & $14(14.4)$ & $24(15.7)$ & $44(4.1)$ & $0(0.0)$ \\
\hline $\begin{array}{l}\text { Exacerbations previous } \\
12 \text { months } \geq 2, \mathrm{n}(\%)\end{array}$ & $1402(51.5)$ & $420(70.2)^{\mathrm{k}}$ & $7(14.0)$ & $104(53.9)$ & $2(12.5)$ & $162(31.5)$ & $13(13.4)$ & - & $356(33.4)^{\mathrm{s}}$ & \\
\hline $\begin{array}{l}\text { Hospitalizations previous } \\
12 \text { months } \geq 1, \text { n (\%) }\end{array}$ & $341(12.5)$ & $162(27.1)^{1}$ & $6(12.0)$ & $81(42.0)$ & $2(12.5)$ & $141(27.4)$ & - & - & $150(14.0)^{\mathrm{t}}$ & - \\
\hline $\begin{array}{l}\text { mMRC dyspnea grade } \\
\geq 2, \mathrm{n}(\%)\end{array}$ & $1526(56.1)$ & $449(64.5)$ & $34(68.0)$ & $150(77.7)$ & $7(43.8)$ & $391(76.1)$ & $39(40.2)$ & $96(62.7)$ & $377(35.2)$ & $8(7.2)$ \\
\hline CAT total score, points & $19.2(8.2)$ & $21.3(8.2)$ & $11.6(7.1)$ & $21.2(7.1)$ & $16.0(10.3)$ & $16.6(7.8)$ & $12.3(7.3)$ & $12.4(7.3)$ & $20.6(8.5)$ & $8.4(6.3)$ \\
\hline CCQ total score, points & - & - & - & - & - & - & - & - & - & - \\
\hline SGRQ total score, points & - & - & - & - & - & $48.0(18.5)^{\circ}$ & $38.3(20.7)$ & $42.1(21.0)^{\mathrm{p}}$ & - & - \\
\hline
\end{tabular}

Values expressed as mean (SD), median (IQR) or number of patients (\%). Abbreviations: BMI= body mass index; CAT= COPD Assessment Test; CCQ= Clinical COPD Questionnaire; DSP= Disease Specific Programme; FEV $=$ Forced Expiratory Volume in the first second; FVC= Forced Vital Capacity; GOLD= Global initiative for chronic Obstructive Lung Disease; LTOT= long-term oxygen therapy; mMRC= modified Medical Research Council dyspnoea scale; SGRQ= St. George's Respiratory Questionnaire.

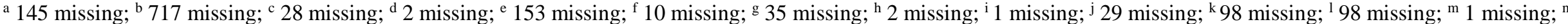

57 missing; ${ }^{\text {o }} 319$ missing; ${ }^{\text {p }} 3$ missing; ${ }^{\mathrm{q}} 62$ missing; ${ }^{\mathrm{r}} 37$ missing; ${ }^{\mathrm{s}} 4$ missing; ${ }^{\mathrm{t}} 1$ missing; ${ }^{\mathrm{u}} 2$ missing. 


\begin{tabular}{|c|c|c|c|c|c|c|c|c|c|c|c|}
\hline & \multicolumn{11}{|c|}{ Dataset resource } \\
\hline Author & $\begin{array}{r}\text { Ringbaek } \\
(69)\end{array}$ & $\begin{array}{r}\text { Da Silva } \\
(18)\end{array}$ & $\begin{array}{r}\text { Da Silva } \\
\text { (19) }\end{array}$ & $\begin{array}{r}\text { Sigari } \\
(70)\end{array}$ & $\begin{array}{r}\text { Tsiligianni } \\
\text { (71) }\end{array}$ & $\begin{array}{r}\text { Tulek } \\
(72)\end{array}$ & $\begin{array}{r}\text { Vestbo } \\
(17)\end{array}$ & $\begin{array}{r}\text { Wilke } \\
\text { (3) }\end{array}$ & $\begin{array}{l}\mathrm{Xie} \\
(73)\end{array}$ & $\begin{array}{r}\text { Yoshikawa } \\
(74)\end{array}$ & $\begin{array}{r}\text { Zogg } \\
(75)\end{array}$ \\
\hline $\begin{array}{lll}\begin{array}{l}\text { Journal } \\
\text { published }\end{array} & \text { and } & \text { year } \\
\end{array}$ & $\begin{array}{r}\text { COPD, } \\
2012\end{array}$ & $\begin{array}{l}\text { Qual Life } \\
\text { Res, } 2014\end{array}$ & $\begin{array}{r}\text { J Bras Pneumol, } \\
2013\end{array}$ & $\begin{array}{r}\text { Rheumatol Int, } \\
2015\end{array}$ & $\begin{array}{r}\text { BMC Pulm Med, } \\
2012\end{array}$ & $\begin{array}{r}\text { Respirology, } \\
2014\end{array}$ & \begin{tabular}{r|r} 
Respir Med, & Respir Med, \\
2014 & 2014
\end{tabular} & J COPD F, 2014 & Chin Med J, 2014 & $\begin{array}{r}\text { Respirology, } \\
2014 \\
\end{array}$ & $\begin{array}{r}\text { BMC Res } \\
\text { Notes, } 2014\end{array}$ \\
\hline Country & Denmark & & Brazil & Iran & Greece & Turkey & $\begin{array}{r}\text { US, France, Germany, Italy, } \\
\text { Spain, UK }\end{array}$ & The Netherlands & Shanghai & Japan & Switzerland \\
\hline Cohort & & & & & & & Adelphi Respiratory DSP & - & - & & \\
\hline $\mathbf{n}$ & 118 & & 50 & 78 & 90 & 119 & 1491 & 698 & 844 & 58 & 68 \\
\hline Gender (male), n (\%) & $47(39.8)$ & & $24(48.0)$ & $45(57.7)$ & $82(91.1)$ & $116(97.5)$ & $1019(68.3)$ & $391(56.0)$ & $659(78.1)$ & $56(96.6)$ & $41(60.3)$ \\
\hline Age, years & $68.2(9.6)$ & & $66.2(8.5)$ & $60.5(8.0)$ & $67.4(8.7)$ & $59.5(9.3)$ & $65.1(10.2)$ & $64.8(8.9)$ & $68.0(9.1)$ & $72.1(9.2)$ & $67.2(10.4)$ \\
\hline Current smoker, n (\%) & $22(18.6)^{\mathrm{a}}$ & & - & 27 (34.6) & $70(77.8)$ & - & $487(32.9)^{\mathrm{d}}$ & $166(23.8)^{g}$ & $623(73.8)$ & $20(34.5)$ & $32(47.1)$ \\
\hline Packyears & $41.7(22.6)^{\mathrm{b}}$ & & - & $29.1(34.7)$ & $60.0(40.0-84.3)$ & $38.3(10.2)$ & $30.0(20.0-45.0)^{\mathrm{e}}$ & $40.0(28.0-50.0)^{\mathrm{h}}$ & $22.5(15.0-31.0)^{\mathrm{k}}$ & $60.0(45.8-80.0)$ & $45.8(32.5)^{\mathrm{n}}$ \\
\hline BMI, kg/m² & $24.8(5.8)$ & & $25.9(5.1)$ & - & $27.8(5.1)$ & $27.6(5.3)$ & $26.5(23.4-29.4)^{\mathrm{f}}$ & $26.1(5.4)$ & $22.9(3.0)$ & $21.0(3.5)$ & $25.1(21.1-28.9)$ \\
\hline FEV $_{1}$ (\% pred.) & $33.7(9.3)$ & & $44.1(13.8)$ & $48.7(17.4)$ & $58.2(18.8)$ & $59.2(20.0)$ & $62.6(17.1)$ & $54.7(22.3)$ & $48.0(17.2)$ & $50.8(19.7)$ & $64.3(21.9)$ \\
\hline $\mathrm{FEV}_{1} / \mathrm{FVC}(\%)$ & - & & $48.7(10.8)$ & $56.1(8.9)$ & $55.7(10.7)$ & - & - & $42.3(14.0)$ & $54.7(10.1)$ & $43.5(12.7)^{1}$ & $50.1(12.4)$ \\
\hline LTOT, n (\%) & $4(3.4)$ & & - & $5(6.4)$ & $1(1.1)$ & - & $117(7.8)$ & $136(19.5)$ & $202(23.9)$ & $10(17.2)$ & $2(3.1)^{\circ}$ \\
\hline GOLD stage, n (\%) - I & $0(0.0)$ & & $0(0.0)$ & $7(9.0)$ & $15(16.7)$ & $17(14.3)$ & $256(17.2)$ & $115(16.5)$ & $31(3.7)$ & $5(8.6)$ & $13(19.1)$ \\
\hline - II & $6(5.1)$ & & $18(36.0)$ & $28(35.9)$ & $46(51.1)$ & $64(53.8)$ & $936(62.8)$ & $259(37.1)$ & $310(36.7)$ & $22(37.9)$ & $38(55.9)$ \\
\hline - III & $70(59.3)$ & & $25(50.0)$ & $35(44.9)$ & $21(23.3)$ & $30(25.2)$ & $241(16.2)$ & $220(31.5)$ & $366(43.4)$ & $25(43.1)$ & $12(17.6)$ \\
\hline- IV & $42(35.6)$ & & $7(14.0)$ & $8(10.3)$ & $8(8.9)$ & $8(6.7)$ & $58(3.9)$ & $104(14.9)$ & $137(16.2)$ & $6(10.3)$ & $5(7.4)$ \\
\hline $\begin{array}{l}\text { Exacerbations previous } \\
12 \text { months } \geq 2, \mathrm{n}(\%)\end{array}$ & - & & - & $31(39.7)$ & $2(2.2)$ & $48(40.3)$ & $465(31.2)$ & $330(47.3)$ & $386(45.7)$ & $7(12.1)$ & $8(11.8)$ \\
\hline $\begin{array}{l}\text { Hospitalizations previous } \\
12 \text { months } \geq 1, \text { n (\%) }\end{array}$ & - & & - & $39(50.0)$ & $5(5.6)$ & $28(23.5)$ & $196(13.1)$ & $239(34.3)$ & $218(25.8)$ & $5(8.6)$ & \\
\hline $\begin{array}{l}\text { mMRC dyspnea grade } \\
\geq 2, \mathrm{n}(\%)\end{array}$ & $118(100.0)$ & & $25(50.0)$ & $70(89.7)$ & $22(24.4)$ & $64(53.8)$ & $758(50.8)$ & $490(70.3)$ & $534(63.3)$ & $36(62.1)$ & $28(41.2)$ \\
\hline CAT total score, points & $18.3(6.6)$ & & $20.8(9.9)$ & $25.1(8.7)$ & $12.9(7.5)$ & $13.1(8.1)$ & $21.0(8.8)$ & $20.0(7.4)$ & $18.3(7.9)$ & $15.2(7.7)$ & $13.3(7.0)$ \\
\hline CCQ total score, points & - & & - & - & $1.6(1.0)$ & - & - & $2.3(1.1)^{\mathrm{i}}$ & - & - & \\
\hline SGRQ total score, points & $42.8(7.0)^{\mathrm{c}}$ & & $44.9(20.4)$ & - & $36.8(18.3)$ & - & - & $54.0(22.0)^{\mathrm{j}}$ & - & $42.0(15.9)^{\mathrm{m}}$ & \\
\hline
\end{tabular}

Values expressed as mean (SD), median (IQR) or number of patients (\%). Abbreviations: BMI= body mass index; CAT= COPD Assessment Test; CCQ= Clinical COPD

Questionnaire; DSP= Disease Specific Programme; $\mathrm{FEV}_{1}=$ Forced Expiratory Volume in the first second; FVC= Forced Vital Capacity; GOLD= Global initiative for chronic

Obstructive Lung Disease; LTOT= long-term oxygen therapy; mMRC= modified Medical Research Council dyspnoea scale; SGRQ= St. George's Respiratory Questionnaire.

a 8 missing; b 13 missing; ${ }^{\text {c }} 113$ missing; ${ }^{\text {d }} 12$ missing; ${ }^{\text {e }} 188$ missing; ${ }^{\text {f }} 44$ missing; ${ }^{\mathrm{g}} 1$ missing; ${ }^{\mathrm{h}} 22 \mathrm{missing} ;{ }^{\mathrm{i}} 3 \mathrm{missing} ;{ }^{\mathrm{j}} 1 \mathrm{missing} ;{ }^{\mathrm{k}} 11 \mathrm{missing} ;{ }^{\mathrm{l}} 1 \mathrm{missing} ;{ }^{\mathrm{m}} 16 \mathrm{missing} ;{ }^{\mathrm{n}} 3$ missing; ${ }^{\circ} 3$ missing. 


\section{RESULTS}

Overall, 337 reports were identified, of which 63 were eligible (Figure 1). Forty-five author groups were able and willing to participate. Finally, 41 datasets were included in the patient level pooled analysis. At the time of inclusion, 3 articles were published with the dataset of the COPD History Assessment In SpaiN (CHAIN) cohort (14), 3 articles used the Adelphi Respiratory Disease Specific Program dataset (one of which is from another subcohort (15) than the other 2 articles $(16,17)), 1$ author group published 2 articles with the same dataset $(18,19)$, and 1 dataset did not have recently measured $\mathrm{FEV}_{1}$ (\% predicted) (5). In addition, the COPD and SYstemic consequences-COmorbidities NETwork (COSYCONET) steering committee approved to share their cohort baseline data (20). Table 1 provides all details per study.

Demographic and clinical characteristics of 18,577 patients with COPD are presented in Table 2. Most patients had a moderate or severe degree of airflow limitation. Spirometric grade 2 was the most prevalent (46\%). Using the GOLD 2017 cut-points, the majority of patients were classified in the high-symptom B/D groups: mMRC, 55.3\%; CAT, 83.6\%; CCQ, 78.8\%; and SGRQ 83.0\%.

The degree of airflow limitation correlated weakly-to-moderately with the mMRC dyspnea grade $(\mathrm{r}=-0.40, \mathrm{P}<.001)$, CAT total score $(\mathrm{r}=-0.26, \mathrm{P}<.001)$, CCQ total score $(\mathrm{r}=-0.37, \mathrm{P}<.001)$, and SGRQ total score $(\mathrm{r}=-0.36, \mathrm{P}<.001$; Figure 2$)$. Moreover, the symptom measures interrelated strongly, with the Pearson product-moment correlation coefficients ranging from 0.540 to 0.799 (all $\mathrm{P}<.001$; Figure 3).

\section{New Cut-Points}

Figure 4 shows the newly proposed cut-points. A CAT cut-point of 18 points, a CCQ cut-point of 1.9 points, and a SGRQ cut-point of 46.0 points reached the highest sensitivity and specificity vs the mMRC dyspnea grade of 2 or higher as point of reference.

\section{Frequency Distribution}

GOLD A/B/C/D frequencies based on current cut-points and the newly derived cut points are shown in Figure 5. Compared with the existing CAT cut-point ( $\geq 10$ points), the new cut-point $(\geq 18$ points) re-classified $30.2 \%$ of the GOLD B/D patients into GOLD A/C. Compared with the existing CCQ cut-point ( $\geq 1$ point), the new cut-point ( $\geq 1.9$ points) re-classified $23.9 \%$ of the GOLD B/D patients into GOLD A/C. Compared to the existing SGRQ cut-point ( $\geq 25$ points), the new cut-point ( $\geq 46$ points) re-classified $34.3 \%$ of the GOLD B/D patients into GOLD A/C. 
Table 2. General characteristics of total population

\begin{tabular}{|c|c|c|c|}
\hline \multicolumn{2}{|l|}{ Variables } & \multicolumn{2}{|l|}{$\mathrm{n}=$} \\
\hline \multicolumn{2}{|l|}{ Gender (male), n (\%) } & 18,577 & $13,370(72 \cdot 0)$ \\
\hline \multicolumn{2}{|l|}{ Age, years } & 18,577 & $66.3(9.6)$ \\
\hline \multicolumn{2}{|l|}{$-\quad 40-50$ years, $\mathrm{n}(\%)$} & & $1,122(6.0)$ \\
\hline \multicolumn{2}{|l|}{ - 51-60 years, n (\%) } & & $3,978(21.4)$ \\
\hline \multicolumn{2}{|l|}{ - $\quad 61-70$ years, $\mathrm{n}(\%)$} & & $6,985(37.6)$ \\
\hline \multicolumn{2}{|l|}{ - $\quad 71-80$ years, n $(\%)$} & & $5,380(29.0)$ \\
\hline \multicolumn{2}{|l|}{ - $\quad>80$ years, $n(\%)$} & & $1,112(6.0)$ \\
\hline \multicolumn{2}{|l|}{ Current smoker, n (\%) } & 16,888 & $6,626(35.7)$ \\
\hline \multicolumn{2}{|l|}{ Pack years } & 14,234 & $38.0(23.0-52.5)$ \\
\hline \multicolumn{2}{|l|}{$\mathrm{BMI}, \mathrm{kg} / \mathrm{m}^{2}$} & 16,934 & $26.5(5.2)$ \\
\hline \multicolumn{2}{|l|}{$\mathrm{FEV}_{1}(\%$ pred.) } & 18,577 & $54.6(19.5)$ \\
\hline \multicolumn{2}{|l|}{$\mathrm{FEV}_{1} / \mathrm{FVC}(\%)$} & 13,692 & $51.8(12.1)$ \\
\hline \multicolumn{2}{|l|}{ LTOT, n $(\%)$} & 12,547 & $1,903(10.2)$ \\
\hline \multirow[t]{4}{*}{ GOLD spirometric grade, n (\%) } & $-\quad 1$ & 18,577 & $2,029(10.9)$ \\
\hline & -2 & & $8,611(46.4)$ \\
\hline & -3 & & $6,026(32.4)$ \\
\hline & -4 & & $1,911(10.3)$ \\
\hline \multicolumn{2}{|c|}{ Exacerbations previous 12 months $\geq 2, \mathrm{n}(\%)$} & 16,607 & $6,443(38.8)$ \\
\hline \multicolumn{2}{|c|}{ Hospitalizations previous 12 months $\geq 1, \mathrm{n}(\%)$} & 13,881 & $2,537(18.3)$ \\
\hline \multirow[t]{5}{*}{ mMRC grade, n (\%) } & & 18,577 & $2,183(11.8)$ \\
\hline & & & $6,122(33.0)$ \\
\hline & & & $5,474(29.5)$ \\
\hline & & & $3,598(19.4)$ \\
\hline & & & $1,200(6.5)$ \\
\hline \multicolumn{2}{|l|}{ mMRC dyspnea grade $\geq 2, \mathrm{n}(\%)$} & 18,577 & $10,272(55.3)$ \\
\hline \multicolumn{2}{|l|}{ CAT total score, points } & 18,577 & $18.4(8.4)$ \\
\hline \multicolumn{2}{|c|}{ Percentage subjects with value $\geq 10, \mathrm{n}(\%)$} & & $15,535(83.6)$ \\
\hline \multicolumn{2}{|l|}{ CCQ total score, points } & 2,047 & $2.1(1.3)$ \\
\hline \multicolumn{2}{|c|}{ Percentage subjects with value $\geq 1, \mathrm{n}(\%)$} & & $1,614(78.8)$ \\
\hline \multicolumn{2}{|l|}{ SGRQ total score, points } & 6,159 & $45.4(20.0)$ \\
\hline \multicolumn{2}{|c|}{ Percentage subjects with value $\geq 25, \mathrm{n}(\%)$} & & $5,114(83.0)$ \\
\hline
\end{tabular}

Values expressed as mean (SD), median (IQR) or number of patients (\%). Abbreviations: BMI= body mass index; $\mathrm{CAT}=\mathrm{COPD}$ Assessment Test; $\mathrm{CCQ}=$ Clinical COPD Questionnaire; $\mathrm{FEV}_{1}=$ Forced Expiratory Volume in the first second; FVC= Forced Vital Capacity; GOLD= Global initiative for chronic Obstructive Lung Disease; LTOT= longterm oxygen therapy; $\mathrm{mMRC}=$ modified Medical Research Council dyspnoea scale; $\mathrm{SGRQ}=$ St. George's Respiratory Questionnaire 

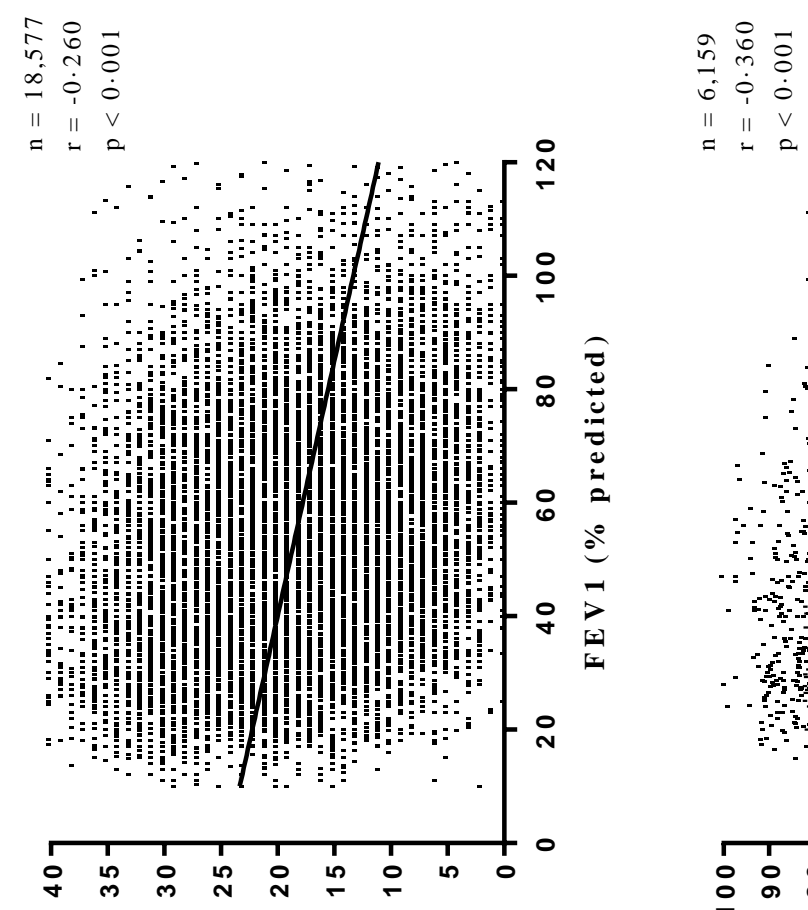

(słu!cd) ว.I oos I



( słu!̣d) ә рв.ı вә
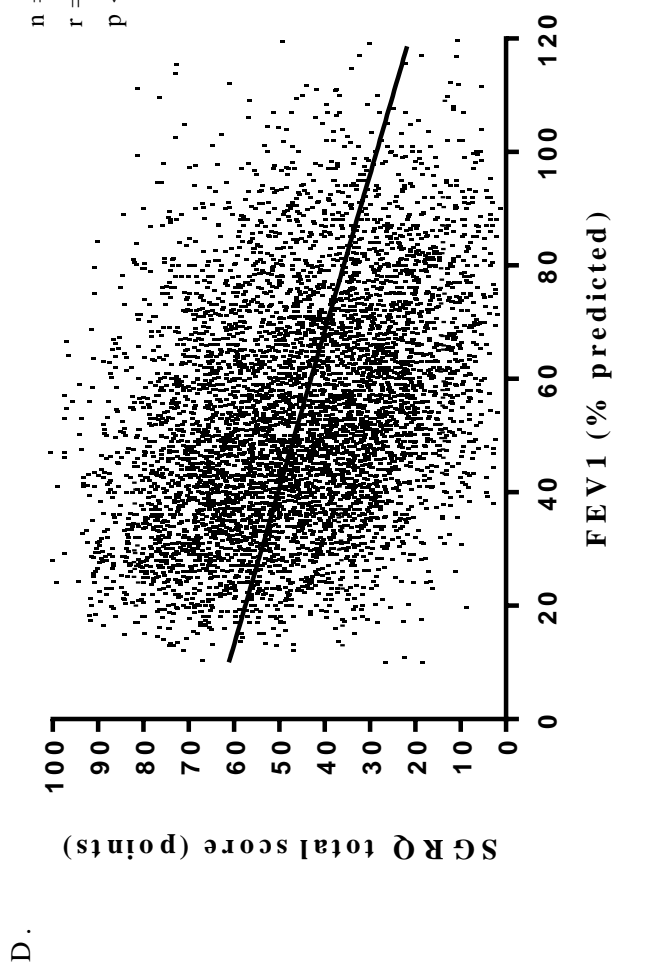

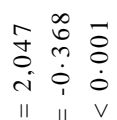

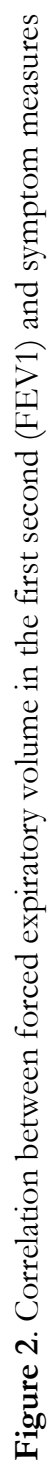

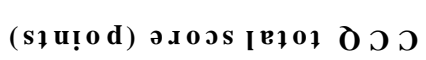



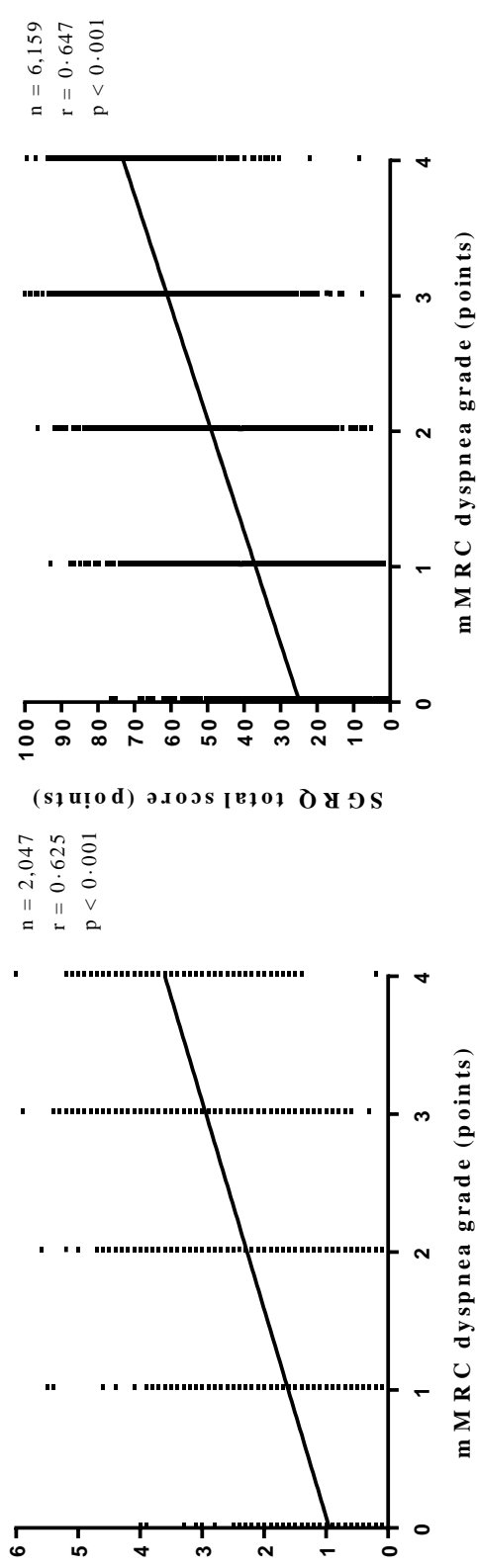

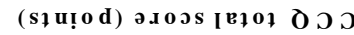

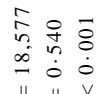

$\begin{array}{lll}\infty & \dot{0} & \dot{0} \\ \| 1 & \| 1 & v \\ \Rightarrow & -1 & 2\end{array}$

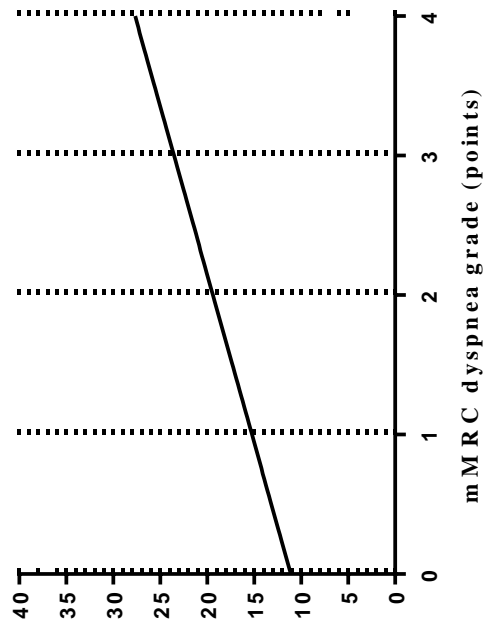

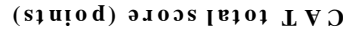

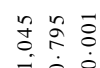



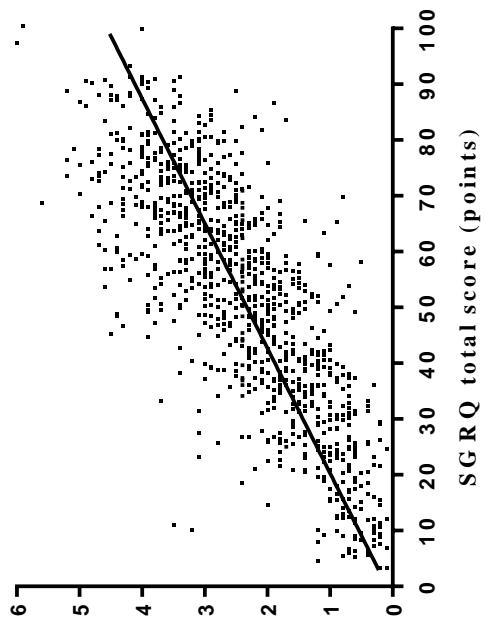

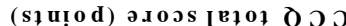

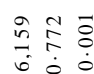

$\begin{array}{lll}0 & 0 & 0 \\ 11 & 11 & V\end{array}$
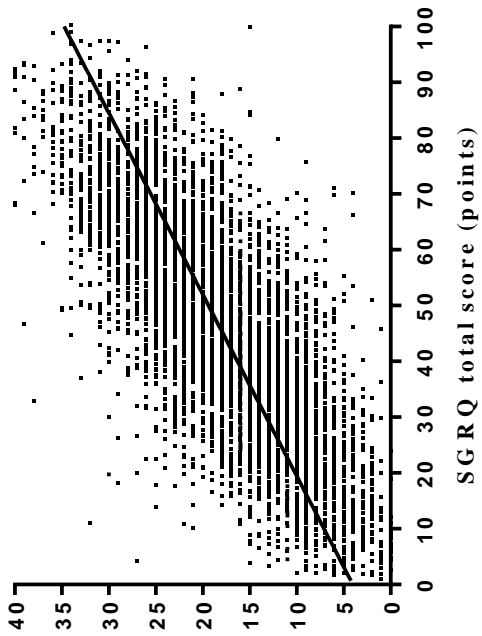



\section{佸起家}

$\begin{array}{lll}\text { i } & 0 & 0 \\ \text { II } & \| & v\end{array}$
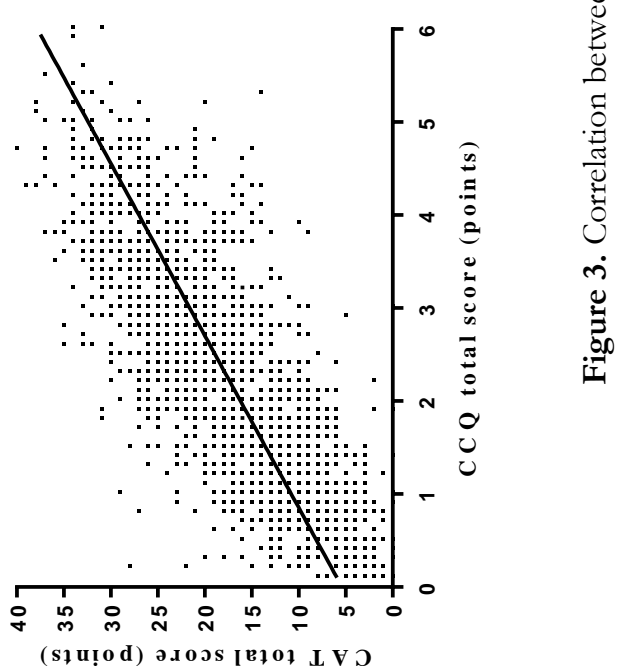
a)

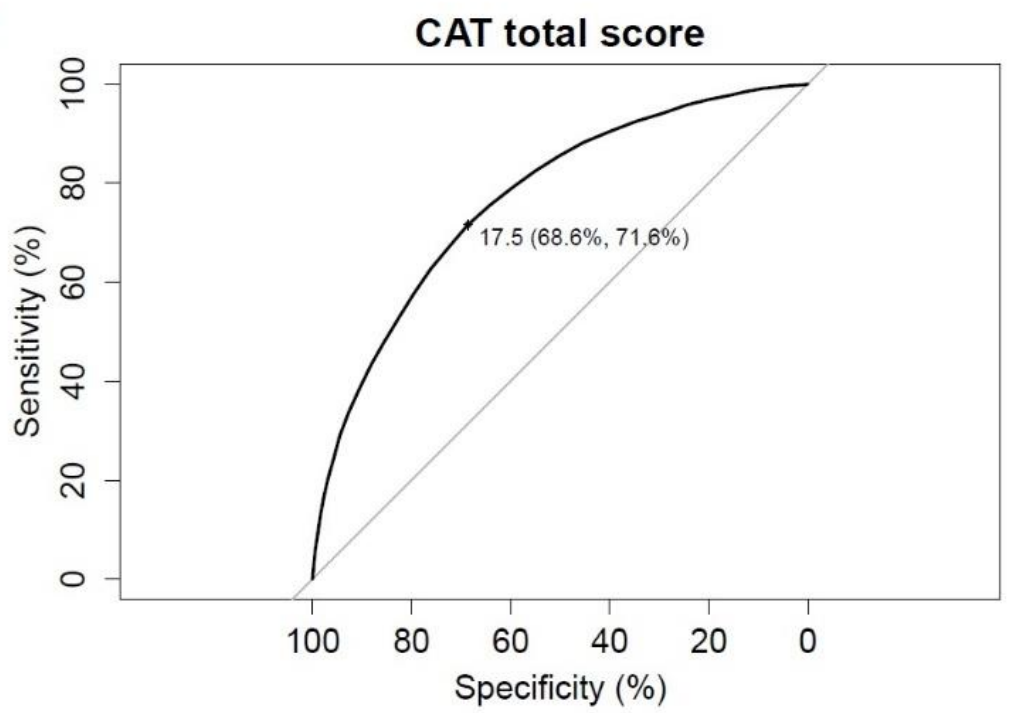

b)

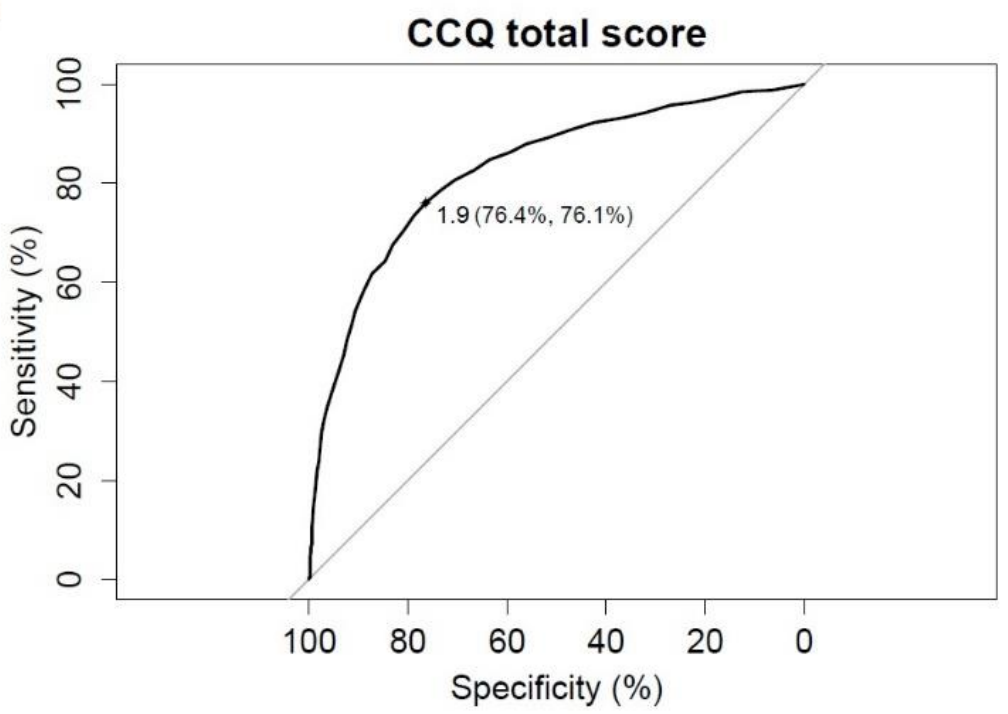

c)

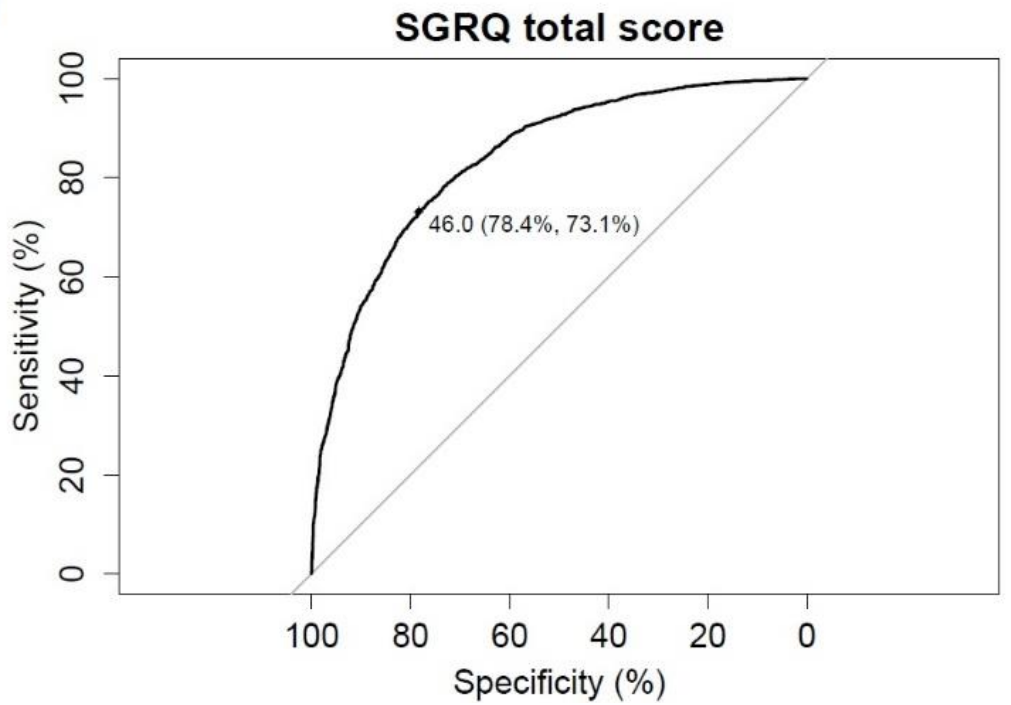

* Optimal cut-point

(specificity, sensitivity) 




\section{DISCUSSION}

Healthcare professionals should be aware of the fact that the choice of symptom measure influences classification, and, in turn, also specific treatment recommendation in patients with COPD. Using $m M R C \geq 2$ points as a reference, a CAT cut-point of 18 points, CCQ cutpoint of 1.9 points, and SGRQ cut-point of 46.0 points reached the highest agreement. Implementation of these newly derived cut-points will influence the management of individual patients and the design and interpretation of clinical studies.

\section{Recommendations}

As the newly derived cut-points reached the highest sensitivity and specificity with the mMRC dyspnea grade of 2 or higher, guidelines committees may need to consider the use of a mMRC dyspnea grade 2 or higher, a CAT total score of 18 points or higher, a CCQ total score of 1.9 points or higher, or a total SGRQ score of 46.0 points or higher to classify patients with COPD as symptomatic (ie, GOLD B or D;

Figure 6). This recommendation is supported by the fact that a CAT total score $\geq 10$ points already occurs in $50 \%$ of current or former smokers without having any airway obstruction (76). The newly derived cut-points enable healthcare professionals to classify the largest proportion of patients into the same GOLD quadrant regardless of their choice of symptom measure.

\section{Clinical Consequences}

Future studies are needed to assess the effectiveness of bronchodilators in COPD patients with and without symptoms, using the newly derived cut-points. For example, GOLD A patients are advised to use short-acting bronchodilators, whereas GOLD B patients are advised to use longacting bronchodilators (1). Therefore, the new cutpoints may reduce the prescription of long-acting bronchodilators in patients who are currently GOLD B, and will become GOLD A by applying the new cut-points. Obviously, the question arises what to do with COPD patients with a mMRC grade below 2 and a CAT score between 10 (current cut-point) and 18 points (newly derived cut point)? This combination of scores suggests that these patients suffer from other symptoms than dyspnea, which can most probably not be treated satisfactorily with the current pulmonary drug therapy.

The newly proposed cut-points may also affect recruitment criteria for upcoming trial designs. Indeed, studies that previously applied the current cut-points, will have an overrepresentation of GOLD B or D patients. Sillen et al (77) showed that there is a lot of heterogeneity in GOLD group $\mathrm{D}$, when applying the existing cutpoints. In turn, adjusting cut-points of the symptom measures to the newly derived cut-points will increase baseline homogeneity of patient populations within observational COPD studies and intervention trials.

The current analysis confirms that the degree of airflow limitation only moderately correlates with the symptom measures. So, the degree of symptom burden cannot accurately be derived from spirometry. Therefore, healthcare professionals need to regularly assess symptoms in patients with COPD. Indeed, a change in symptom scores may even have a prognostic value in patients with COPD (78). 


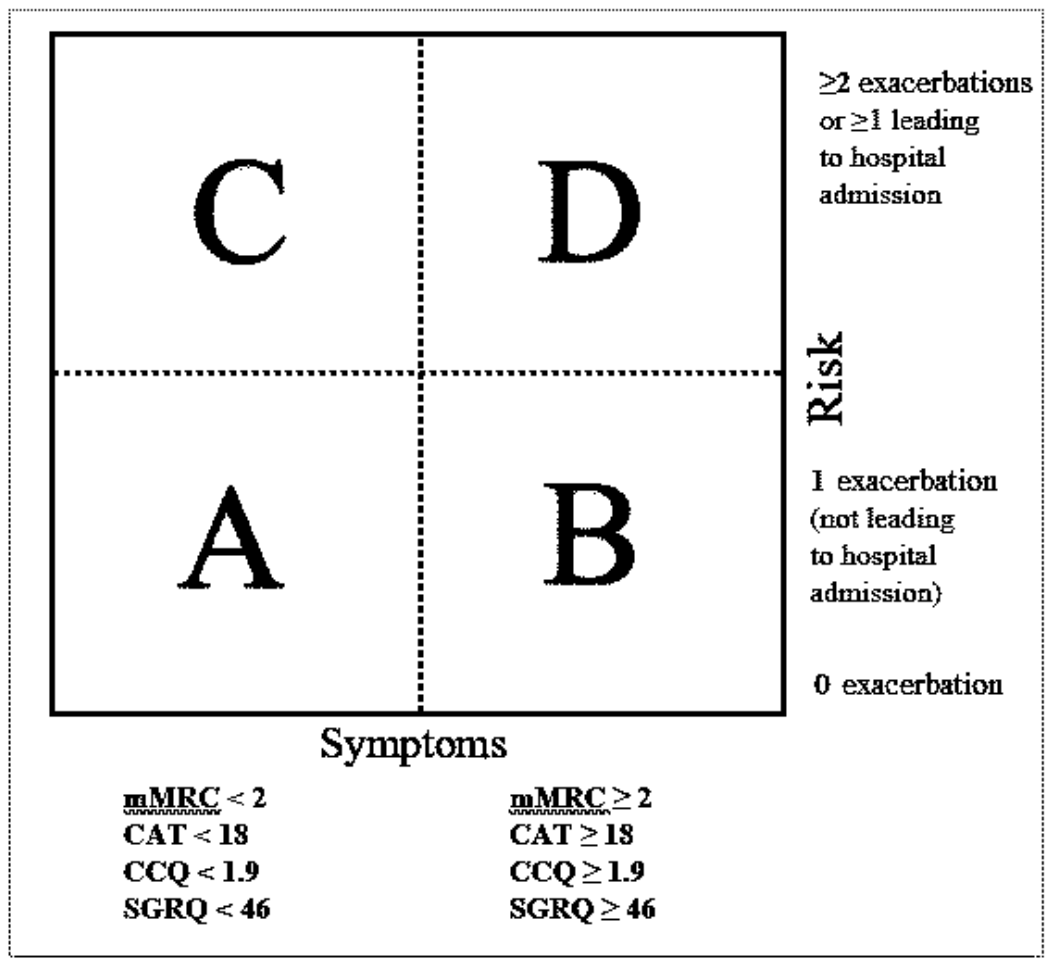

Figure 3. Suggested GOLD ABCD diagram, using the new cut-points to assess symptoms. CAT $=\mathrm{COPD}$ Assessment Test; $\mathrm{CCQ}=$ Clinical COPD Questionnaire; $\mathrm{GOLD}=$ Global initiative for chronic Obstructive Lung Disease; $\mathrm{mMRC}=$ modified Medical Research Council dyspnoea scale; SGRQ= St. George's Respiratory Questionnaire.

\section{Strengths and Limitations}

The pooled, multicenter, multinational, patient level dataset with a large number of patients and global coverage is a major strength. Indeed, this resulted in a heterogeneous sample of patients with COPD, also including a high number of patients with a low mMRC dyspnea grade (grade 0 : 2183 patients; grade 1: 6122 patients), patients with a mild degree of airflow limitation (spirometric grade 1: 2029 patients), and 1,122 patients younger than 50 years of age. Moreover, patients were recruited from various care settings (ie, primary care, general population, hospital outpatients). This makes the results more generalizable.

A limitation of the current study is that the largest proportion of patients was male $(72.0 \%)$. Although this seems to over-represent the male sex, it is probably a reliable representation of the current COPD population in the participating cohorts (79). Less data were available for the CCQ total score (2047 patients) and SGRQ total score (6159 patients). Furthermore, the definition of COPD, current, former or never smoker and the definition of exacerbations and hospitalizations could differ between studies. Finally, the mMRC dyspnea grade solely captures symptoms of dyspnea, which may, together with spirometry and history of exacerbations/hospitalizations, be a suitable guidance for treatment recommendations. Nevertheless, mMRC dyspnea scale may be too limited to truly understand the impact of COPD. Indeed, symptoms like fatigue, pain and insomnia, may also occur in patients with COPD (80). Therefore, CAT, CCQ, or SGRQ may be preferred to more broadly characterize the daily symptoms of patients with COPD. Obviously, when CAT, CCQ, and SGRQ are applied for the binary classification of high vs low symptoms, there will still be discrepancy between these symptom measures. So, the GOLD Scientific Committee may want to consider the choice of 1 symptom measure or applying the worst scoring questionnaire to classify patients into groups A/C or B/D. 


\section{CONCLUSIONS}

To objectively define a symptom burden score equivalent to a mMRC dyspnea grade of 2 or higher, a CAT total score of $\geq 18$ points, a CCQ total score of $\geq 1.9$ points, or a SGRQ total score of $\geq 46$ points should be used. Following this grading, about one-third of the patients in GOLD groups $\mathrm{B} / \mathrm{D}$ are re-classified to GOLD groups A/C. This implies that guidelines committees may consider adapting our evidence-based cut-points of symptom measures. 


\section{REFERENCES}

1. Vogelmeier CF, Criner GJ, Martinez FJ, et al. Global strategy for the diagnosis, management, and prevention of chronic obstructive lung disease 2017 report: GOLD executive summary. AJRCCM; 2017.

2. Jones PW, Adamek L, Nadeau G, et al. Comparisons of health status scores with MRC grades in COPD: Implications for the GOLD 2011 classification. Eur Respir J; 2013;42:647-654.

3. Wilke S, Smid DE, Spruit MA, et al. The 2014 updated GOLD strategy: A comparison of the various scenarios. JCOPDF; 2014;2:212-220.

4. Casanova C, Marin JM, Martinez-Gonzalez C, et al. Differential effect of Mmrc dyspnea, CAT and CCQ for symptom evaluation within the new GOLD staging and mortality in COPD. Chest 2015;159:159168.

5. Holt S, Sheahan D, Helm C, et al. Little agreement in GOLD category using CAT and mMRC in 450 primary care COPD patients in New Zealand. NPJ Prim Care Respir Med 2014;24:14025.

6. Agusti A, Hurd S, Jones P, et al. FAQs about the GOLD 2011 assessment proposal of COPD: A comparative analysis of four different cohorts. Eur Respir J 2013;42: 1391e1401.

7. Lee CH, Lee J, Park YS, et al. Chronic obstructive pulmonary disease (COPD) assessment test scores corresponding to modified Medical Research Council grades among COPD patients. Korean J Intern Med 2015;30:629-637.

8. Tsiligianni IG, Alma HJ, de Jong C, et al. Investigating sensitivity, specificity, and area under the curve of the Clinical COPD Questionnaire, COPD Assessment Test, and Modified Medical Research Council scale according to GOLD using St George's Respiratory Questionnaire cutoff 25 (and 20) as reference. Int J Chron Obstruct Pulmon Dis 2016;11:1045-1052.

9. Kon SS, Canavan JL, Nolan CM, et al. The clinical chronic obstructive pulmonary disease questionnaire: Cut point for GOLD 2013 classification. Am J Respir Crit Care Med 2014;189:227-228.

10. Vogelmeier CF, Vestbo J, Hurd SS, et al. Changes in GOLD: Today and tomorrow. Lancet Respir Med 2015;3:424-426.

11. Jones PW, Harding G, Berry P, et al. Development and first validation of the COPD Assessment Test. Eur Respir J 2009;34:648-654.

12. Pickard AS, Yang Y, Lee TA. Comparison of health-related quality of life measures in chronic obstructive pulmonary disease. Health Qual Life Outcomes 2011;9:26.

13. Youden WJ. Index for rating diagnostic tests. Cancer 1950;3:32-35.

14. Lopez-Campos JL, Peces-Barba G, Soler-Cataluna JJ, et al. Chronic obstructive pulmonary disease history assessment in Spain: A multidimensional chronic obstructive pulmonary disease evaluation. Study methods and organization. Arch Bronconeumol 2012;48:453-459.

15. Price D, West D, Brusselle G, et al. Management of COPD in the UK primary-care setting: An analysis of real-life prescribing patterns. Int J Chron Obstruct Pulmon Dis 2014;9:889-904.

16. Jones PW, Nadeau G, Small M, et al. Characteristics of a COPD population categorised using the GOLD framework by health status and exacerbations. Resp Med 2014;108:129-135.

17. Vestbo J, Vogelmeier C, Small M, et al. Understanding the GOLD 2011 Strategy as applied to a realworld COPD population. Resp Med 2014;108:729-736.

18. da Silva GF, Morano MT, Sales MP, et al. Comparison of face-to-face interview and telephone interview administration of COPD assessment test: A randomized study. Qual Life Res 2014;23:1193-1197.

19. da Silva GP, Morano MT, Viana CM, et al. Portuguese-language version of the COPD Assessment Test: Validation for use in Brazil. J Bras Pneumol 2013;39: 402e408.

20. Karch A, Vogelmeier C, Welte T, et al. The German COPD cohort COSYCONET: Aims, methods and descriptive analysis of the study population at baseline. Resp Med 2016;114:27-37.

21. Han J, Dai L, Zhong N, Young D. Breathlessness or health status in chronic obstructive pulmonary disease: The impact of different definitions. COPD 2015; 12:115e125.

22. Grzelewska-Rzymowska I, Patora-Mikolajczyk J, Gorski P. Stratification of patients with COPD according to the 2011 GOLD report. Pneumonologia i Alergologia Polska 2014;82:415-421. 
23. Zhang R, Tan X, He Q, et al. Comparison of symptom and risk assessment methods among patients with chronic obstructive pulmonary disease. Chin Med J 2014;127:2594-2598.

24. Paulin LM, Diette GB, Blanc PD, et al. Occupational exposures are associated with worse morbidity in patients with chronic obstructive pulmonary disease. Am J Respir Crit Care Med 2015;191:557-565.

25. Gimeno-Santos E, Raste Y, Demeyer H, et al. The PROactive instruments to measure physical activity in patients with chronic obstructive pulmonary disease. Eur Respir J 2015;46:988-1000.

26. Khurana S, Ravi A, Sutula J, et al. Clinical characteristics and airway inflammation profile of COPD persistent sputum producers. Respir Med 2014;108: 1761-1770.

27. Kurt OK, Tosun M, Kurt EB, Talay F. Pentraxin 3 as a novel biomarker of inflammation in chronic obstructive pulmonary disease. Inflammation 2015; 38:89-93.

28. Dal Negro RW, Bonadiman L, Turco P. Sensitivity of the COPD assessment test (CAT questionnaire) investigated in a population of 681 consecutive patients referring to a lung clinic: The first Italian specific study. Multidiscip Respir Med 2014;9:15.

29. Rieger-Reyes C, Garcia-Tirado FJ, Rubio-Galan FJ, Marin-Trigo JM. Classification of chronic obstructive pulmonary disease severity according to the new Global Initiative for Chronic Obstructive Lung Disease 2011 guidelines: COPD assessment test versus modified Medical Research Council scale. Arch Bronconeumol 2014;50:129-134.

30. Hwang YI, Jung KS, Lim SY, et al. A Validation Study for the Korean Version of Chronic Obstructive Pulmonary Disease Assessment Test (CAT). Tuberc Respir Dis 2013;74:256-263.

31. Zhang Y, Tu YH, Fei GH. The COPD assessment test correlates well with the computed tomography measurements in COPD patients in China. Int J Chron Obstruct Pulmon Dis 2015;10:507-514.

32. Stoll P, Ulrich M, Bratke K, et al. Imbalance of dendritic cell co-stimulation in COPD. Respir Res 2015;16:19.

33. Nowak C, Sievi NA, Clarenbach CF, et al. Accuracy of the hospital anxiety and depression scale for identifying depression in chronic obstructive pulmonary disease patients. Pulmon Med 2014;2014: 973858.

34. Lopes AJ, Mafort TT. Correlations between small airway function, ventilation distribution, and functional exercise capacity in COPD patients. Lung 2014;192: 653-659.

35. Ohno T, Wada S, Hanada S, et al. Efficacy of indacaterol on quality of life and pulmonary function in patients with COPD and inhaler device preferences. Int J Chron Obstruct Pulmon Dis 2014;9:107-114.

36. Okutan O, Tas D, Demirer E, Kartaloglu Z. Evaluation of quality of life with the chronic obstructive pulmonary disease assessment test in chronic obstructive pulmonary disease and the effect of dyspnea on disease-specific quality of life in these patients. Yonsei Med J 2013;54:1214-1219.

37. Bai P, Sun Y, Jin J, et al. Disturbance of the OPG/RANK/RANKL pathway and systemic inflammation in COPD patients with emphysema and osteoporosis. Respir Res 2011;12:157.

38. Zhou QT, Mei JJ, He B, et al. Chronic obstructive pulmonary disease assessment test score correlated with dyspnea score in a large sample of Chinese patients. Chin Med J 2013;126:11-15.

39. Agusti A, Soler-Cataluna JJ, Molina J, et al. Does the COPD assessment test (CAT(TM)) questionnaire produce similar results when self- or interviewer administered? Qual Life Res 2015;24:2345-2354.

40. Billington J, Coster S, Murrells T, Norman I. Evaluation of a nurse-led educational telephone intervention to support self-management of patients with chronic obstructive pulmonary disease: A randomized feasibility study. COPD 2015;12:395-403.

41. Boutou AK, Tanner RJ, Lord VM, et al. An evaluation of factors associated with completion and benefit from pulmonary rehabilitation in COPD. BMJ Open Respir Res 2014;1:e000051.

42. de Torres JP, Marin JM, Martinez-Gonzalez C, et al. Clinical application of the COPD assessment test: Longitudinal data from the COPD History Assessment in Spain (CHAIN) cohort. Chest 2014;146:111122.

43. Casanova C, Marin JM, Martinez-Gonzalez C, et al. New GOLD classification: Longitudinal data on group assignment. Respir Res 2014;15:3. 
44. Chaplin E, Gibb M, Sewell L, Singh S. Response of the COPD Assessment Tool in stable and postexacerbation pulmonary rehabilitation populations. J Cardiopulmon Rehabil Prevent 2015;35:214218.

45. Dodd JW, Hogg L, Nolan J, et al. The COPD assessment test (CAT): Response to pulmonary rehabilitation. A multicentre, prospective study. Thorax 2011;66: 425-429.

46. Horita N, Yomota M, Sasaki M, et al. Evaluation of the chronic obstructive pulmonary disease assessment test in Japanese outpatients. Clin Respir J 2013.

47. Jehn M, Donaldson G, Kiran B, et al. Tele-monitoring reduces exacerbation of COPD in the context of climate changeeA randomized controlled trial. Environ Health 2013;12:99.

48. Kelly JL, Bamsey O, Smith C, et al. Health status assessment in routine clinical practice: The chronic obstructive pulmonary disease assessment test score in outpatients. Respiration 2012;84:193-199.

49. Kim S, Oh J, Kim YI, et al. Differences in classification of COPD group using COPD assessment test (CAT) or modified Medical Research Council (mMRC) dyspnea scores: A cross-sectional analyses. BMC Pulmon Med 2013;13:35.

50. Kon SS, Dilaver D, Mittal M, et al. The Clinical COPD Questionnaire: Response to pulmonary rehabilitation and minimal clinically important difference. Thorax 2014;69:793-798.

51. Kwon N, Amin M, Hui DS, et al. Validity of the COPD assessment test translated into local languages for Asian patients. Chest 2013;143:703-710.

52. Lee SD, Huang MS, Kang J, et al. The COPD assessment test (CAT) assists prediction of COPD exacerbations in high-risk patients. Respir Med 2014;108:600-608.

53. Ladeira I, Gomes T, Castro A, et al. The overall impact of COPD (CAT) and BODE index on COPD male patients: Correlation? Revista Portuguesa de Pneumologia 2015;21:11-15.

54. Lopez-Campos JL, Fernandez-Villar A, Calero-Acuna C, et al. Evaluation of the COPD Assessment Test and GOLD patient types: A cross-sectional analysis. Int J Chron Obstruct Pulmon Dis 2015;10:975-984.

55. Manca S, Rodriguez E, Huerta A, et al. Usefulness of the CAT, LCOPD, EQ-5D and COPDSS scales in understanding the impact of lung disease in patients with alpha-1 antitrypsin deficiency. COPD 2014;11:480-488.

56. Maricic L, Vceva A, Visevic R, et al. Assessment of endothelial dysfunction by measuring von Willebrand factor and exhaled nitric oxide in patients with chronic obstructive pulmonary disease. Collegium antropologicum 2013;37: 1153-1160.

57. Mendoza L, Horta P, Espinoza J, et al. Pedometers to enhance physical activity in COPD: A randomised controlled trial. Eur Respir J; 2015:45347-45354.

58. Mihaltan F. "Inspirom"eOverview of the relationship between the pulmonologist and the COPD patient in Romania. Pneumologia 2015;64:24-28.

59. Miravitlles M, Barrecheguren M, Roman-Rodriguez M. Frequency and characteristics of different clinical phenotypes of chronic obstructive pulmonary disease. Int J Tuberc Lung Dis 2015;19:992-998.

60. Miravitlles M, Molina J, Quintano JA, et al. Factors associated with depression and severe depression in patients with COPD. Respir Med 2014;108: 1615-1625.

61. Minami S, Yamamoto S, Ogata Y, et al. Ambulatory pulse oximetry monitoring in Japanese COPD outpatients not receiving oxygen therapy. Multidisciplinary Respir Med 2014;9:24.

62. Nakken N, Janssen DJ, van den Bogaart EH, et al. An observational, longitudinal study on the home environment of people with chronic obstructive pulmonary disease: The research protocol of the Home Sweet Home study. BMJ Open 2014;4:e006098.

63. Nishijima Y, Minami S, Yamamoto S, et al. Influence of indacaterol on daily physical activity in patients with untreated chronic obstructive pulmonary disease. Int J Chron Obstruct Pulmon Dis 2015;10:439444.

64. Novotna B, Koblizek V, Zatloukal J, et al. Czech multicenter research database of severe COPD. Int J Chron Obstruct Pulmon Dis 2014;9:1265-1274.

65. Pothirat C, Kiatboonsri S, Chuchottaworn C. Validation of the new COPD assessment test translated into Thai in patients with chronic obstructive pulmonary disease. BMC Pulmon Med 2014;14:193. 
66. Pothirat C, Chaiwong W, Phetsuk N, et al. Dialectal influence on chronic pulmonary disease assessment test: The reliability and validity study. Int J Chron Obstruct Pulmon Dis 2015;10:541-548.

67. Price DB, Baker CL, Zou KH, et al. Real-world characterization and differentiation of the Global Initiative for Chronic Obstructive Lung Disease strategy classification. Int J Chron Obstruct Pulmon Dis 2014;9:551-561.

68. Raghavan N, Lam YM, Webb KA, et al. Components of the COPD Assessment Test (CAT) associated with a diagnosis of COPD in a random population sample. COPD 2012;9:175-183.

69. Ringbaek T, Martinez G, Lange P. A comparison of the assessment of quality of life with CAT, CCQ, and SGRQ in COPD patients participating in pulmonary rehabilitation. COPD 2012;9:12-15.

70. Sigari N, Moghimi N, Shahraki FS, et al. Anti-cyclic citrullinated peptide (CCP) antibody in patients with wood-smoke-induced chronic obstructive pulmonary disease (COPD) without rheumatoid arthritis. Rheumatol Int 2015;35:85-91.

71. Tsiligianni IG, van der Molen T, Moraitaki D, et al. Assessing health status in COPD. A head-to-head comparison between the COPD assessment test (CAT) and the clinical COPD questionnaire (CCQ). BMC Pulmon Med 2012; 12:20.

72. Tulek B, Atalay NB, Yildirim G, et al. Cognitive function in chronic obstructive pulmonary disease: Relationship to global initiative for chronic obstructive lung disease 2011 categories. Respirology 2014;19:873-880.

73. Xie G, Zhang Y, Zhou X. New disease severity classification of patients with stable chronic obstructive pulmonary disease in Shanghai. Chin Med J 2014; 127:3046e3050.

74. Yoshikawa M, Fujita Y, Yamamoto Y, et al. Mini-Nutritional Assessment Short-Form predicts exacerbation frequency in patients with chronic obstructive pulmonary disease. Respirology 2014;19:1198-1203.

75. Zogg S, Durr S, Miedinger D, et al. Differences in classification of COPD patients into risk groups AD: A cross-sectional study. BMC Res Notes 2014;7:562.

76. Woodruff PG, Barr RG, Bleecker E, et al. Clinical significance of symptoms in smokers with preserved pulmonary function. N Engl J Med 2016;374: 1811-1821.

77. Sillen MJ, Franssen FM, Delbressine JM, et al. Heterogeneity in clinical characteristics and comorbidities in dyspneic individuals with COPD GOLD D: Findings of the DICES trial. Resp Med 2013;107:11861194.

78. Wilke S, Jones PW, Mullerova H, et al. One-year change in health status and subsequent outcomes in COPD. Thorax 2015;70:420-425.

79. Buist AS, McBurnie MA, Vollmer WM, et al. International variation in the prevalence of COPD (the BOLD Study): A population-based prevalence study. Lancet 2007;370:741-750.

80. Janssen DJ, Spruit MA, Uszko-Lencer NH, et al. Symptoms, comorbidities, and health care in advanced chronic obstructive pulmonary disease or chronic heart failure. J Palliat Med 2011;14:735-743. 